\title{
Subarachnoid Hemorrhage and Prostatic Involvement in Granulomatosis with Polyangiitis - An Atypical Presentation
}

\author{
Raymond Bak Hei Chu ${ }^{1, \star}$, Priscilla Ching Han Wong ${ }^{2}$, Joey Ka Ming Wai ${ }^{1}$, Florence Hiu Yi Yap ${ }^{1}$ \\ ${ }^{1}$ Department of Anaesthesia and Intensive Care, Prince of Wales Hospital, Shatin, New Territories, Hong Kong \\ ${ }^{2}$ Division of Rheumatology, Department of Medicine and Therapeutics, Prince of Wales Hospital, Shatin, New Territories, Hong Kong
}

\begin{abstract}
Granulomatosis with Polyangiitis (GPA) is a form of Anti-neutrophil cytoplasmic antibody (ANCA)-associated vasculitis. We report a case of a 56-year-old man with GPA who first presented with acute urinary retention, and then developed pulmonary cavitating lesions and subarachnoid hemorrhage (SAH). The SAH was due to a ruptured right vertebral artery aneurysm, which was subsequently embolized. The patient initially had a good response to immunosuppressive therapy but later succumbed due to fulminant sepsis. SAH is a rare complication of GPA and usually not associated with a detectable aneurysm. Our patient is the second case in the literature where an aneurysm had been detected on angiography and the first case where examination of the culprit aneurysm revealed features of vasculitis.
\end{abstract}

Keywords: Wegener's Granulomatosis (WG); Granulomatosis with Polyangiitis (GPA); Anti-neutrophil Cytoplasmic Autoantibody (ANCA); Subarachnoid Hemorrhage (SAH); Intracranial Hemorrhage (ICH); Neurological; Urological; Prostate; Retention of Urine; Pulmonary; Cavitation.

\section{INTRODUCTION}

Granulomatosis with Polyangiitis (GPA) (formerly known as Wegener's granulomatosis) is a form of Anti-neutrophil cytoplasmic autoantibody (ANCA)associated vasculitis involving the small and mediumsized vessels. Classically, the disease is a triad of upper airway, lungs and kidney vasculitis. However, all other organ systems, including the prostate and central nervous system, can also be involved. Here, we report a patient with atypical presentation of GPA which posed significant diagnostic challenges.

\section{CASE PRESENTATION}

A 56-year-old Chinese male, with history of recurrent first metatarsophalangeal joint (MTPJ) arthritis refractory to low purine diet and gout medication, first presented with acute retention of urine requiring urinary catheterization. On palpation, his prostate was large and irregular while the serum prostate specific antigen was $3.99 \mathrm{ng} / \mathrm{ml}$ (normal $<4 \mathrm{ng} / \mathrm{ml}$ ). He was discharged home with the urinary catheter in situ. A bone scintigraphy later showed no evidence of bony metastases.

Three weeks later, he was admitted to the hospital for dry cough and constitutional symptoms including fever, night sweats, and weight loss. On admission, the white cell count was $16.7 \times 10^{9} / \mathrm{L}$ with $81 \%$ neutrophils, $7 \%$ lymphocytes, and $3 \%$ eosinophils, while the C-reactive protein was $166 \mathrm{mg} / \mathrm{l}$ and erythrocyte sedimentation rate was $118 \mathrm{~mm} / \mathrm{hr}$. On chest X-ray (CXR), he had a left upper lobe mass of $5 \mathrm{~cm}$ in diameter with cavitation. CT of the thorax revealed a loculated fluid collection in the posterior segment of the left upper lobe, as well as other small fluid collections, on a background of emphysematous changes.

The working diagnosis was multiple infective lung abscesses so he was given amoxicillin-clavulanate and then piperacillin-tazobactam. Serial CT of the thorax showed interval enlargement of the suspected lung abscesses (Figure 1) and fever was persistent. Ultrasound-guided aspiration of the left upper lobe

( 2019 by the Hong Kong Society of Rheumatology and World Scientific Publishing Co. Pte. Ltd. Ð Open Access article under the CC BY-NC-ND license (http://creativecommons.org/licenses/by-nc-nd/4.0/).

Received 19 June 2019; Accepted 25 June 2019; Published 8 July 2019

${ }^{*}$ Corresponding author: Raymond B. H. Chu, Department of Anaesthesia and Intensive Care, 4/F Main Clinical Block and Trauma Centre, Prince of Wales Hospital, Shatin, New Territories, Hong Kong, E-mail: drcbhr@gmail.com 
Figure 1. CT thorax showing progressive enlargement of cavitatory lesions on days 2, 12, and 25 (from left to right).

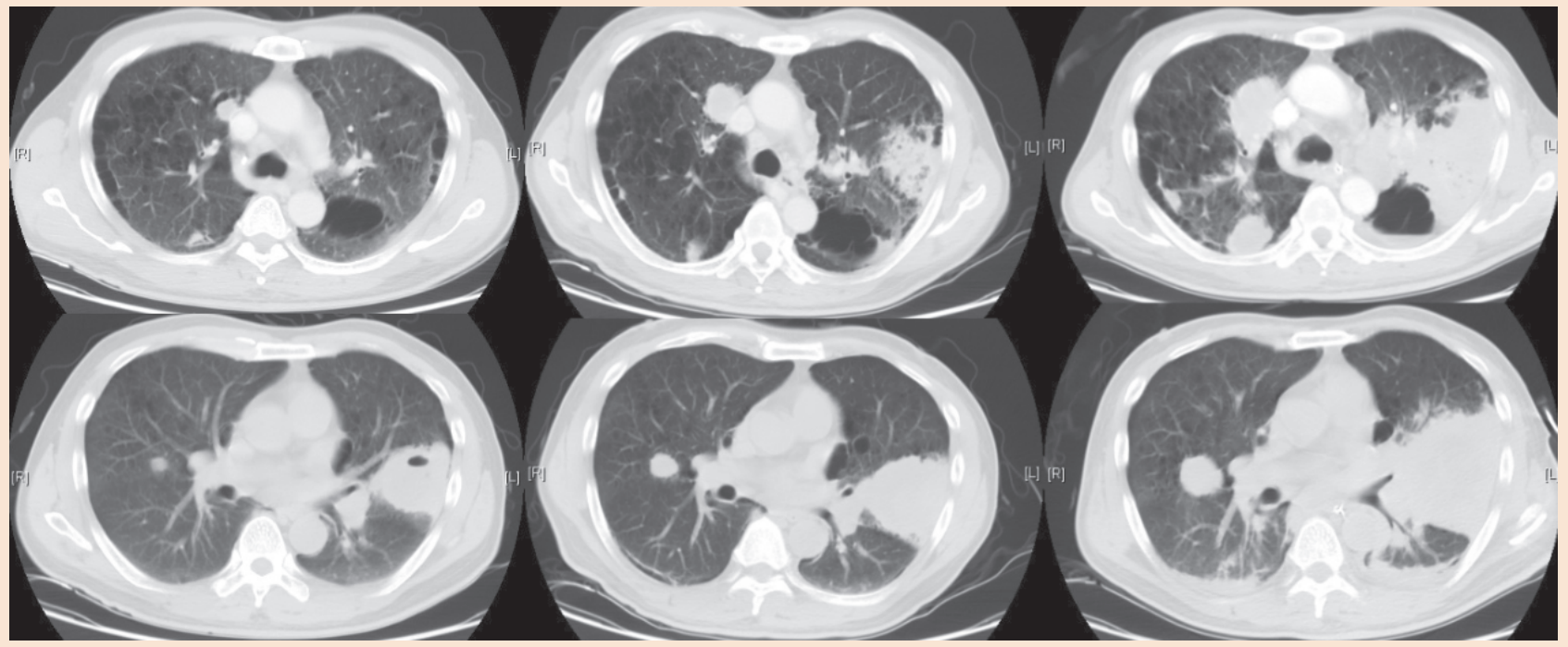

Figure 2. CT pelvis showing progressive enlargement of prostatic mass on days 12 and 22 (from left to right).

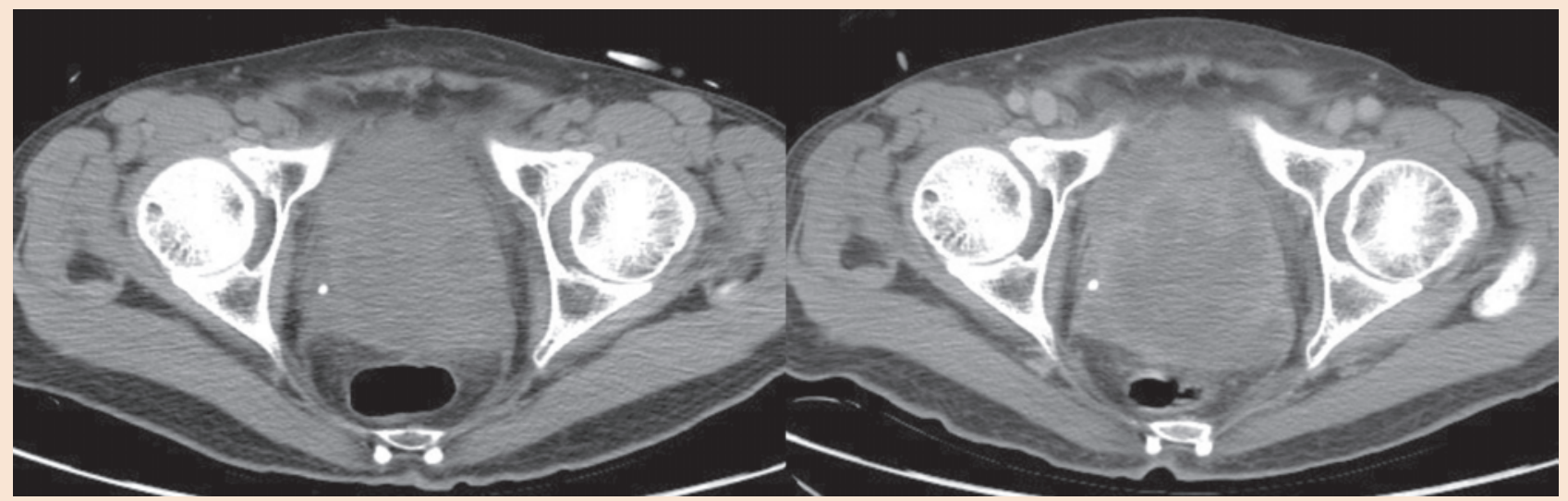

lung abscess was repeated four times. Microbiological investigations of the abscess aspirates, blood, sputum, bronchoalveolar lavage, and urine for bacteria, fungus, and mycobacterium were all negative. On day 15 , a USG-guided needle biopsy of the left lung lesion was performed. The lung biopsy revealed suppurative granulomatous inflammation and suppurative necrotic material focally rimmed by histiocytes and multinucleated giant cells. Histochemical staining did not show any microorganisms. Antibiotics were further escalated empirically to meropenem, vancomycin and micafungin.
The patient failed to wean off the urinary catheter, and required suprapubic catheter insertion on day 10 for difficult urethral catheterization. Serial CT of the pelvis revealed a multi-loculated rim-enhancing cystic prostatic mass with progressive enlargement (Figure 2). Transrectal ultrasound guided (TRUS) aspiration of the cystic mass was attempted and the pus grew carbapenem resistant Enterobacteriaceae (Escherichia coli), Candida albicans, Bifidobacterium species, and Bacteroides species, and antibiotics were changed to tigecyline. On day 26, a transurethral incision and drainage of the suspected prostate abscess was performed. The biopsy 
Figure 3. CT brain showing acute subarachnoid and intraventricular hemorrhage with hydrocephalus (left) and right vertebral angiography showing right vertebral artery aneurysm (right).

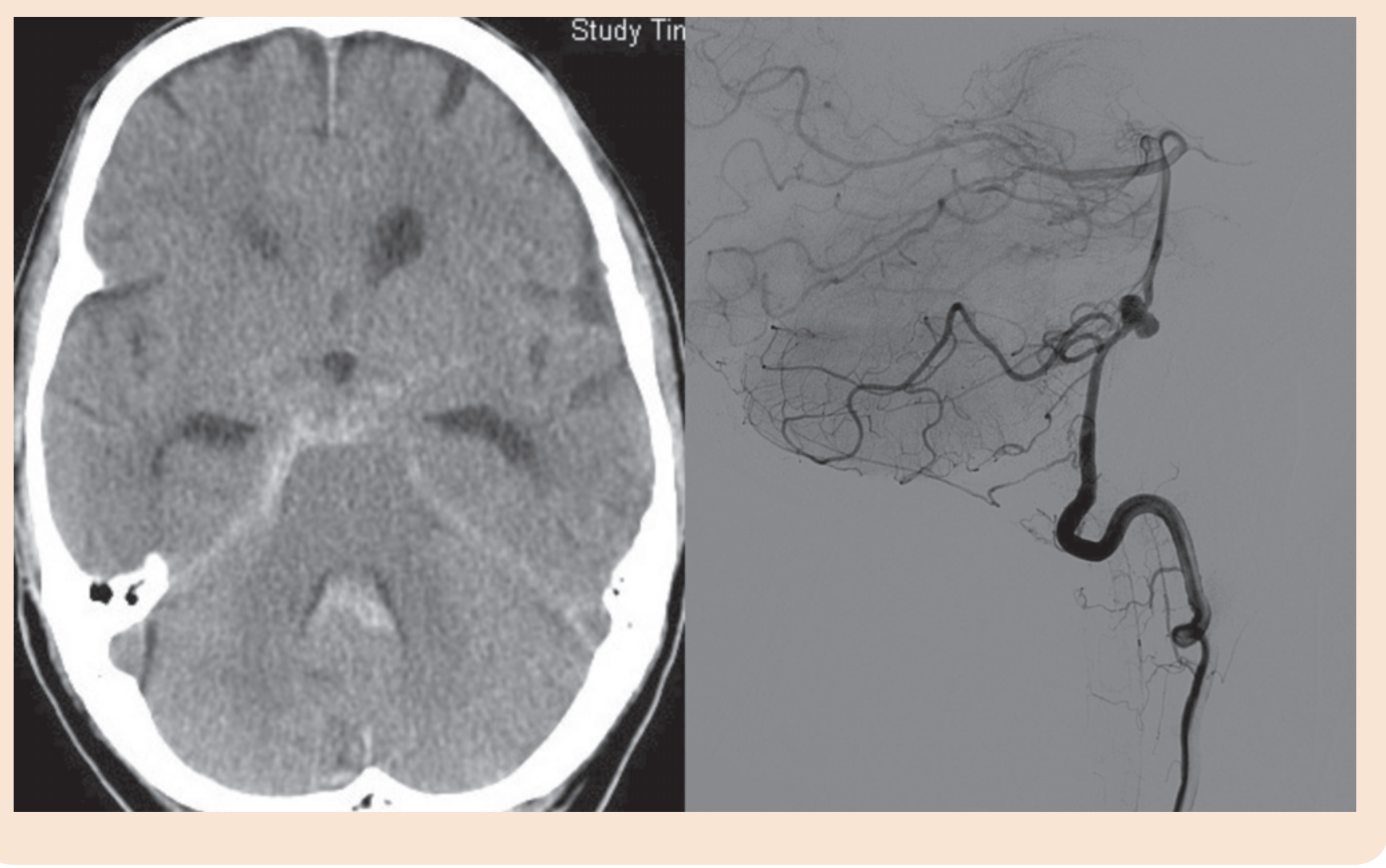

specimen demonstrated necrotizing granulomatous inflammation with necrotizing granulomas rimmed by epithelioid histiocytes.

On day 24, the patient had an acute deterioration in conscious state requiring airway protection. Urgent CT brain revealed acute subarachnoid and intraventricular hemorrhage with early hydrocephalus (Figure 3). CT angiography of the cerebral arteries located a ruptured saccular aneurysm with lobulated appearance arising from the right vertebral artery. He underwent emergent external ventricular drain (EVD) insertion followed by vertebral angiography and embolization to the right vertebral artery aneurysm (Figure 3).

By the third week, serum antibody screen was done and was positive for c-ANCA. The anti-protinase 3 (PR3) antibody level was strongly positive, > $200 \mathrm{RU} /$ $\mathrm{ml}$, but anti-myeloperoxidase antibody level was within normal range, 7.4 RU/ml. The diagnosis of GPA was suspected and further investigations were done to look for other organ involvement.

Review of the CT brain showed features of frontal and ethmoidal sinusitis. Nasopharyngoscopy noted small ulcerative patches with dilated capillaries over the left lateral nasal wall and right posterior septum. Retinoscopy revealed signs of vasculitis, Roth's spots, as well as Terson's syndrome. The patient also developed maculopapular rashes over bilateral palms and soles, and skin biopsy of the right hand dorsum exhibited leukocytoclastic vasculitis. Ultrasound of his swollen right big toe revealed significant first MTPJ synovial hypertrophy. Synovial biopsy demonstrated extensive necrosis and vague granuloma without features of gouty tophi. Serum creatinine was $90 \mathrm{umol} / \mathrm{L}$ while estimated glomerular filtration rate was $82.8 \mathrm{ml} / \mathrm{min}$ (by MDRD equation corrected for body surface area). There was proteinuria with urine total protein-to-creatinine ratio of $1.74 \mathrm{mg} / \mathrm{mg}$ creatinine (normal $<0.2 \mathrm{mg} / \mathrm{mg} \mathrm{Cr}$, nephrotic range $>3.5 \mathrm{mg} / \mathrm{mg} \mathrm{Cr}$ ) but urine microscopy showed neither dysmorphic red blood cells nor red cell casts.

On the fourth week, the diagnosis of GPA was established in this patient based on a combination of positive ANCA and PR3 serology, multisystem involvement and histological evidence of necrotizing 
Figure 4. AP chest X-ray on days 29 and 39, before and after pulse steroids respectively (from left to right).

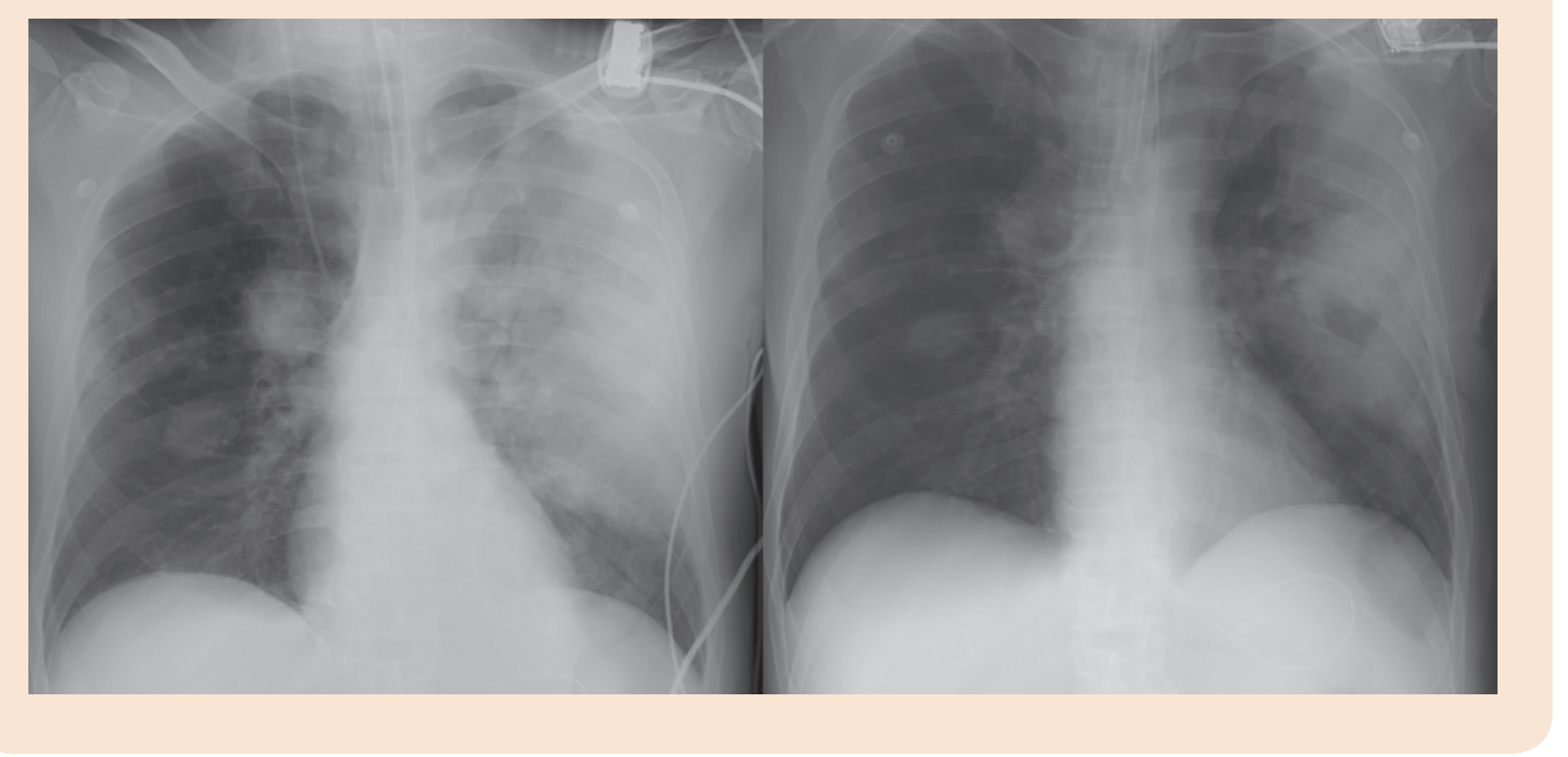

vasculitis. According to the American College of Rheumatology criteria for GPA diagnosis, our patient presented with two of the four criteria, namely abnormal CXR and histological evidence of granulomatous inflammation (Sensitivity 88\%, Specificity 92\%). On day 29 , the patient was started on intravenous methylprednisolone one gram daily for three doses, followed by prednisolone $65 \mathrm{mg}$ daily. Fever subsided after the pulse steroids and serial CXR showed resolving infiltrates (Figure 4). He awoke to obey commands after weaning sedation.

Unfortunately, one week after initiating steroid therapy, the patient deteriorated with high fever, septic shock and worsening conscious state. Septic work-up was done, the external ventricular drain was removed, and meropenem was started. Repeat CT brain revealed diffuse cerebral edema and he rapidly progressed to brain death. In view of the poor neurological prognosis, the patient's family agreed to withdraw life support and he succumbed on day 39 of hospital admission. Subsequently, cultures from the blood, cerebrospinal fluid and EVD all grew extended-spectrum $\beta$-lactamase producing Klebsiella, which was only intermediately sensitive to meropenem.

Post-mortem examination of vertebral artery and brain were performed. Sections of the brain exhibited features of meningitis with dense neutrophilic infiltrates in the meninges. There was vasculitis in the right vertebral artery featuring disruption of the internal elastic lamina, fibrinoid necrosis of the vascular wall associated with nuclear dusts, neutrophilic and histiocytic infiltrates. No well formed granuloma was seen. Histochemical stain for fungus and bacteria were negative. The CD163 stain highlighted the abundant histiocytes at the internal elastic lamina. Though secondary vasculitis of the vertebral artery due to meningitis could not be differentiated from primary vasculitis based on morphology, the overall picture was compatible with GPA.

\section{DISCUSSION}

In the central nervous system, GPA most commonly affects the cranial nerves but can also cause intracranial hemorrhage and cerebral vascular thrombosis [2,6]. Such manifestations range from $1.7-26.7 \%$ of GPA cases (Table 1). In a review of 104 patients, Drachman [2] found $54 \%$ of patients had some form of neurological involvement while 9\% had developed cerebrovascular events. In another review of 324 patients by Nishino et al. [10], 33.6\% of patients had neurological involvement while $4 \%$ had cerebrovascular events. It has been postulated that these cerebrovascular events occur as a result of vasculitis of the nervous system [2].

Subarachnoid hemorrhage is a rare complication of GPA with uncertain etiology and we have identified nine other cases (Table 2). Most of these GPA patients are of male gender. Brain autopsy was performed in only three cases, of which two showed vasculitis $[1,2,6]$. As GPA 
Table 1. Review on clinical manifestations of patients with GPA.

\begin{tabular}{llllll}
\hline Study & Total & \multicolumn{4}{l}{ Organ involvement (\%) } \\
\cline { 5 - 7 } & patients & Joint & PNS $^{\dagger}$ & CNS $^{\ddagger}$ & Renal \\
\hline Drachman (1963) [2] & 104 & - & 54 & 54 & - \\
Hoffman et al. (1992) [9] & 158 & 67 & 15 & 8 & 77 \\
Nishino et al. (1993) [10] & 324 & - & 33.6 & 33.6 & - \\
Huong et al. (1995) [11] & 80 & 45 & 23 & 8 & 58 \\
Reinhold-Keller et al. (2000) [12] & 155 & 77 & 40 & 11 & 70 \\
Stone (2003) [13] & 180 & 66.1 & 7.2 & 1.7 & 68 \\
Holle et al. (2011) [14] & 445 & 73 & 23 & 10 & 59.1 \\
Mahr et al. (2013) [15] & 396 & - & 28.3 & 28.3 & - \\
Sharma et al. (2018) [16] & 105 & 43.8 & 11.4 & 26.7 & 51.4 \\
\hline$\dagger$ PNS - Peripheral nervous system, $\neq$ CNS - Central nervous system. & & &
\end{tabular}

Table 2. Case reports of subarachnoid hemorrhage.

\begin{tabular}{lllll}
\hline Reference & Age & Sex & Cerebral angiogram & Outcome \\
\hline Tuhy et al. (1958) [1] & 39 & Male & Not done & Died \\
Drachman (1963) [2] & 30 & Male & Not done & Died \\
Rordam and Hansen (1971) [3] & 53 & Male & Not done & Died \\
Venning et al. (1991) [4] & 50 & Male & Negative & Survived \\
& 36 & Male & Negative & Survived \\
Cruz and Segal (1997) [5] & 71 & Male & Negative & Survived \\
Nardone et al. (2004) [6] & 78 & Female & Not done & Died \\
Takei et al. (2004) [7] & 34 & Male & Left anterior choroidal & Survived \\
& & & artery aneurysm & \\
Miles et al. (2011) [8] & 74 & Female & Not done & Died \\
Present case & 56 & Male & Right vertebral artery & Died \\
& & & aneurysm & \\
\hline
\end{tabular}

typically involves small- to medium-sized blood vessels, detection of the culprit aneurysm is often difficult. Our patient is the second case where an aneurysm had been successfully identified on angiography. $\mathrm{He}$ is also the first patient in the literature where examination of the culprit aneurysm revealed vasculitis, although without granulomas.

As joint involvement by GPA affects $43.8-67 \%$ of patients (Table 1), we postulate that his MTPJ arthritis was an early sign of disease. Prostatic involvement by GPA, being the most common example of urogenital involvement, is quoted to range from $2.3-7.4 \%[17,18]$.
Patients typically present with chronic obstructive symptoms, hematuria, recurrent urinary tract infections, or acute urinary retention [11,19-21]. These symptoms occur at the first presentation $26 \%$ of the time. These patients often have elevated PSA and radiological lung changes, therefore are frequently mistaken to have metastatic prostate cancer [21]. Renal involvement is common and ranges from $51.4-77 \%$ (Table 1). Our patient had proteinuria but no significant renal impairment. Unfortunately, no renal biopsy was done.

Our patient presented significant diagnostic difficulty and reminds us that systemic vasculitis can 
often mimic infection with persistent negative cultures. A misdiagnosis of sepsis could lead to unnecessary use of prolonged broad-spectrum antibiotics with emergence of antibiotic resistance. Pulse steroids remain the mainstay of treatment for severe GPA, rendering these patients at high risk of infections. Our patient initially had a good response to steroids, but eventually died from fulminant EVD-related intracranial infection with ventriculitis and bacteremia, caused by multi-drug resistant Klebsiella. EVD is frequently necessary in neurological ICU patients. However, nosocomial EVDrelated infections are a significant cause of morbidity and mortality.

In conclusion, we presented a patient with GPA who developed prostatism, multiple lung lesions and SAH. Systemic vasculitis often poses challenges in diagnosis and management. It is important to recognize ANCAassociated vasculitis in view of their poor prognosis and the need for early treatment.

\section{ACKNOWLEDGEMENTS}

We thank Dr. Paul CL Choi (Department of Anatomical and Cellular Pathology, Prince of Wales Hospital) for his interpretation of the cerebral histopathological specimens.

Conflict of interest: The authors declare no conflict of interest.

Informed consent: Written informed consent was obtained from the patient reported in the study.

Funding: No funding was acquired for this study.

\section{REFERENCES}

[1] Tuhy JE, Maurice GL, Niles NR. Wegener's granulomatosis. Am J Med. 1958;25:638-46.

[2] Drachman DA. Neurologic involvement in Wegener's granulomatosis. Arch Neurol. 1963;8:145-55.

[3] Rordam P, Hansen LA. Wegener's granulomatosis: report of a case running a protracted course with discussion of therapeutic possibilities. Dan Med Bull. 1971;18:43-6.

[4] Venning MC, Burn DJ, Bashir SH, Deopujari CE, Mendelow AD. Subarachnoid haemorrhage in Wegener's granulomatosis, with negative four vessel angiography. Br J Neurosurg. 1991;5(2):195-8.

[5] Cruz DN, Segal AS. A patient with Wegener's granulomatosis presenting with a subarachnoid hemorrhage: case report and review of CNS disease associated with Wegener's granulomatosis. Am J Nephrol. 1997;17:181-6.
[6] Nardone R, Lochner P, Tezzon F. Wegener's granulomatosis presenting with intracerebral hemorrhages. Cerebrovasc Dis. 2004;17:81-82.

[7] Takei H, Komaba Y, Kitamura H, Hayama N, Osawa H, Furukawa T, Hasegawa O, Iino Y, Katayama Y. Aneurysmal subarachnoid haemorrhage in a patient with Wegener's granulomatosis. J Clin Exp Nephro. 2004;8(3):274-8.

[8] Miles J, McWilliams L, Liu W, Preston D. Subarachnoid hemorrhage in Wegener granulomatosis: a case report and review of the literature. CNS Spectrums. 2011;16(5):121-6.

[9] Hoffman G, Kerr G, Leavitt R, et al. Wegener's granulomatosis: an analysis of 158 patients. Ann Intern Med. 1992;116:488-98.

[10] Nishino H, Rubino FA, DeRemee RA, Swanson JW, Parisi JE. Neurological involvement in Wegener's granulomatosis: an analysis of 324 consecutive patients at the Mayo Clinic. Ann Neurol. 1993;33:4-9.

[11] Huong DL, Papo T, Piette JC, Wechsler B, Bletry O, Richard F, Godeau P. Urogenital manifestations of Wegener granulomatosis. Medicine. 1995;74(3):152-61.

[12] Reinhold-Keller E, Beuge N, Latza U, et al. An interdisciplinary approach to the care of patients with Wegener's granulomatosis. Long-term outcome in 155 patients. Arthritis Rheum. 2000; 43:1021-32.

[13] Stone JH. Wegener's Granulomatosis Etanercept Trial Research Group. Limited versus severe Wegener's granulomatosis: baseline data on patients in the Wegener's granulomatosis etanercept trial. Arthritis Rheum. 2003;48:2299-309.

[14] Holle JU, Gross WL, Latza U, Nölle B, Ambrosch P, Heller M, Fertmann R, Reinhold-Keller E. Improved outcome in 445 patients with Wegener's granulomatosis in a German vasculitis center over four decades. Arthritis \& Rheumatism. 2011;63: 257-66.

[15] Mahr A, Katsahian S, Varet H, et al. Revisiting the classification of clinical phenotypes of anti-neutrophil cytoplasmic antibodyassociated vasculitis: a cluster analysis. Ann Rheum Dis. 2013;72:1003-10.

[16] Sharma A, Naidu GSRSNK, Rathi M, et al. Clinical features and long term outcomes of 105 granulomatosis with polyangiitis patients: a single centre experience from north India. Int J Rheum Dis. 2018;21:278-84.

[17] Stillwell TJ, DeRemee RA, McDonald TJ, Weiland LH, Engen DE. Prostatic involvement in Wegener's granulomatosis. J Urol. 1987;138(5):1251-3.

[18] Walton EW. Giant-cell granuloma of the respiratory tract (Wegener's granulomatosis). Br Med J. 1958;2(5091):265-70.

[19] Gaber KA, Ryley NG, Macdermott JP, Goldman JM. Wegener's granulomatosis involving prostate. Urology. 2005;66(1):195.

[20] Alba MA, Moreno-Palacios J, Beça S, Cid MC. Urologic and male genital manifestations of granulomatosis with polyangiitis. Autoimmun Rev. 2015;14:897-902.

[21] Yatsyshyn R, Zimba O, Bahrii M, Doskaliuk B, Huryk V. Prostate involvement in granulomatosis with polyangiitis. Rheumatol Int. 2019;39(7):1269-77. 